\title{
Situación de salud mental en Colombia
}

\author{
Mental health situation in Colombia
}

Carlos A. Palacio A. ${ }^{1 *}$

${ }^{1}$ Facultad de medicina, Universidad de Antioquia, Medellín, Colombia.

*Corresponding author: cpalacio@udea.edu.co

Entendida la salud de un ser humano con el estado de bienestar integral, con la pretensión de una buena calidad para los individuos; es innegable que el componente de salud mental en el mundo ha estado relegado por elementos de orden social y cultural. La Organización Mundial de la Salud (OMS) en las últimas dos décadas ha priorizado este componente, intentando trazar políticas desde la promoción de la salud con la estrategia de estilos de vida saludables, la prevención intentando controlar y minimizar el riesgo y los determinantes sicológicos y sociales de afectación en la salud mental; mejorar el acceso, la oportunidad y la calidad de la atención de los eventos prioritarios de alteración en la salud mental y por último fomentando actividades de rehabilitación en quienes a consecuencia de estos eventos tienen secuelas.

En nuestra región, Latinoamérica, los sistemas de salud se encuentran en crisis. La financiación de un sistema equitativo y justo es complejo, la contribución estatal a la salud no es suficiente; en los sistemas mixtos el régimen contributivo es menor que el subsidiado y tampoco es el necesario, y en los sistemas privados terminan generando brechas que son luego imposibles de subsanar. Es necesario, que las políticas de salud mental en un país deriven en acciones articuladas, que den cuenta de dos prioridades: la prevención primaria, con estrategias de promoción y prevención poblacionales que lleven a impactar y logren mantener el bienestar y la buena calidad de vida de un buen porcentaje de las comunidades; el segundo un desarrollo coherente del componente de atención primaria en salud (APS) que brinde acceso, oportunidad y calidad en la atención de los eventos mentales. Es importante resaltar, cuando se habla de salud mental, hacer énfasis en el problema de estigmatización de ella, cultural y socialmente en nuestra región sigue estando la salud mental, tanto en la condición de sujeto que requiere acompañamiento como el de talento humano que trabaja en el área, como una condición discriminada, peyorativa o relegada a un segundo plano. Los procesos de sensibilización y educación continúan siendo necesarios para desmontar el tabú que existe. Es común encontrar en gran parte de nuestros individuos las dificultades de reflexionar, analizar y decidir la búsqueda de ayuda cuando se encuentra una alteración del orden mental en él o en personas cercanas. Carecemos de capacidades y competencias sencillas y básicas para una adecuada adaptación a situaciones vitales que enfrentamos en el día a día, conflictos relaciones, familiares o laborales, que con la sola mediación de la palabra y capacidad de escucha pueden resolverse, una buena tolerancia a la frustración, resilencia, para continuar a pesar de los obstáculos o negativas que encontremos. Pero tampoco tenemos competencias para conocer cuando se presentan signos o síntomas que están mostrándonos presencia de alteraciones mentales.

Trastornos tan comunes como los de ansiedad y del animo, no son reconocidos por quienes los sufren y mas grave aún ni por el personal de salud. Las cifras, son escandalosas, el $60 \%$ de las personas que acuden a un servicios de atención básica en salud, tienen síntomas ansiosos y depresivos, solo el $30 \%$ de ellos se les indaga y diagnostican estas alteraciones, pero un porcentaje menor recibe el tratamiento adecuado. Estamos hablando, que apenas un pequeño porcentaje de personas que presentan alguna alteración mental tienen tratamiento adecuado. Las consecuencias son dramáticas, los indicadores de salud siguen muy de bajo de lo esperado, incluso el indicador de mortalidad se ve afectado por problemas como la autoagresión y la heteroagresión.

La conducta suicida se ha convertido en un verdadero problema de salud mental en el mundo, sin duda derivado de los problemas anteriormente mencionados y de otros adicionales que la acompañan. Datos tan preocupantes, como que en el mundo se presenta un suicidio por minuto, o tasas de suicidio según regiones desde el 8 al 100 por 100.000. habitantes; mientras que los intentos de suicidio son 10 a 20 veces mas que los suicidios, ambas con consecuencias graves para quienes presentan esta conducta como para quienes les rodean. 
En diferentes programas mundiales de gestión del riesgo para este evento en salud se ha observado su eficacia reduciendo la incidencia y las consecuencias graves de manera importante. La prevención de factores determinantes sico-sociales con programas pertinentes y eficientes, además involucrar la atención en el primer nivel con el componente de atención primaria de quienes presenten la conducta con abordajes interdisciplinarios e intervenciones de familia. Para ejemplo, el $60 \%$ de las personas que se suicidan tienen trastorno depresivo, si se crean procesos para mejorar la accesibilidad y la oportunidad en la atención de las personas que lo sufren estaríamos impactando el determinante mas importante para este evento.

En conclusión, el camino esta trazado, construir y trabajar por un modelo integrado de atención en salud que construya verdaderas rutas de atención integral en salud para las diferentes patologías prevalentes, con un buen sistema de referencia y contrareferencia, valiéndonos de tecnologías y estrategias que optimicen su eficiencia. 